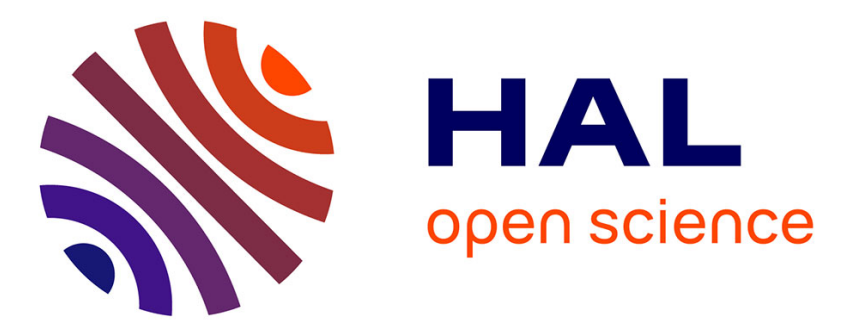

\title{
Dilution Effects in a Spin Crossover System, Modelled in Terms of Direct and Indirect Intermolecular Interactions
}

H. Constant-Machado, J. Linares, F. Varret, J. Haasnoot, J. Martin, J. Zarembowitch, A. Dworkin, Azzedine Bousseksou

\section{- To cite this version:}

H. Constant-Machado, J. Linares, F. Varret, J. Haasnoot, J. Martin, et al.. Dilution Effects in a Spin Crossover System, Modelled in Terms of Direct and Indirect Intermolecular Interactions. Journal de Physique I, 1996, 6 (9), pp.1203-1216. 10.1051/jp1:1996124 • jpa-00247241

\section{HAL Id: jpa-00247241 https://hal.science/jpa-00247241}

Submitted on 1 Jan 1996

HAL is a multi-disciplinary open access archive for the deposit and dissemination of scientific research documents, whether they are published or not. The documents may come from teaching and research institutions in France or abroad, or from public or private research centers.
L'archive ouverte pluridisciplinaire HAL, est destinée au dépôt et à la diffusion de documents scientifiques de niveau recherche, publiés ou non, émanant des établissements d'enseignement et de recherche français ou étrangers, des laboratoires publics ou privés. 


\title{
Dilution Effects in a Spin Crossover System, Modelled in Terms of Direct and Indirect Intermolecular Interactions
}

H. Constant-Machado $\left({ }^{1, *}\right)$, J. Linares $\left({ }^{1}\right)$, F. Varret $\left({ }^{1, * *}\right)$, J.G. Haasnoot $\left({ }^{2}\right)$, J.P.Martin $\left({ }^{3}\right)$, J. Zarembowitch $\left({ }^{3}\right)$, A. Dworkin $\left({ }^{4}\right)$ and A. Bousseksou $\left({ }^{5}\right)$

$\left({ }^{1}\right)$ Laboratoire de Magnétisme et d'Optique $\left({ }^{* *}\right)$, Université de Versailles, 78035 Versailles Cedex, France

$\left({ }^{2}\right)$ Institute of Chemistry, Gorlaeus Laboratorıes, Leiden University, POB 9502, 2300 RA Leiden, The Netherlands

$\left({ }^{3}\right)$ Laboratoire de Chimie Inorganique $\left({ }^{* * *}\right)$, Université Parıs Sud, 91405 Orsay Cedex, France

$\left({ }^{4}\right)$ Laboratoire de Chimie Physique des Matériaux Amorphes $\left({ }^{* * * *}\right)$, Université Parıs Sud, 91405 Orsay Cedex, France

$\left({ }^{5}\right)$ Laboratoire de Chimie de Coordination $\left({ }^{* * * * * *}\right), 31077$ Toulouse Cedex, France

(Received 21 July 1995, revised 27 February 1996, received in final form 7 May 1996, accepted 10 May 1996)

PACS.31.70.Ks - Molecular solids

PACS.33.45.+x - Mössbauer spectra

PACS.64.60.-i - General studies of phase transitions

\begin{abstract}
We have studied by ${ }^{57} \mathrm{Fe}$ Mössbauer spectroscopy and magnetic measurements the spin transition exhibited by the serıes: $\left[\mathrm{Fe}_{x} \mathrm{Co}_{1-x}\left(4,4^{\prime}-b i s-1,2,4 \text {-triazole }\right)_{2}(\mathrm{NCS})_{2}\right] . \mathrm{H}_{2} \mathrm{O}$, consisting of a spin-crossover rron(II) system diluted in a high-spin cobalt(II) matrix. We performed Monte Carlo simulations of the thermal variation of the high-spin fraction, using a two-level Ising-type Hamiltonian. A good agreement with experiment is obtained by the introduction of an indirect interaction of the spin-crossover units via the non-spin crossover unit, the origin of which is discussed in sterıc terms.
\end{abstract}

\section{Introduction}

The physical nature of the cooperative interactions responsible for the abrupt transition in spin-crossover solids is still a subject of discussion. So far, macroscopic descriptions in terms of regular solutions [1] or in terms of elastic theory [2] have succeeded in explaining most of the characteristics of spin transitions: for example the conversion curve $n_{\mathrm{HS}}(T)$, where $n_{\mathrm{HS}}$ is the high-spin fraction, as well as $\Delta H$ and $\Delta S$, which respectively are the changes in enthalpy and entropy associated with the conversion of a mole of the spin-crossover unit.

$\left(^{*}\right)$ On leave from Universidad Central de Venezuela

$\left({ }^{* *}\right)$ Author for correspondence (e-mall: francors.varret@physique.uvsq.fr)

$\left({ }^{* * *}\right)$ URA 1531 CNRS

$\left({ }^{* * * *}\right)$ URA 420 CNRS

$\left({ }^{* * * * *}\right)$ URA 1104 CNRS

$\left({ }^{* * * * *}\right)$ UPR 8241 CNRS

(C) Les Éditions de Physıque 1996 
Here we focus on the microscopic description of the problem. We previously introduced an Ising-like model [3], adapted from previous works [4,5], which was shown to be equivalent, in the mean-field approach, to the regular solution model in the Bragg- Williams approximation [5]. Also, a microscopic counterpart of the elastic model was recently proposed [6], and was solved for the $1 \mathrm{D}$ case.

The Ising-like model is a two-level model including phenomenological Ising interactions. The two levels have different energies (gap $\Delta$ ) and degeneracies (ratio $g=g_{\mathrm{HS}} / g_{\mathrm{LS}}$ ); it is formally equivalent to a true Ising system under a temperature-dependent field [7]. The Hamiltonian is:

$$
\hat{H}=\Sigma \frac{\Delta}{2} \hat{\sigma}_{\imath}-\Sigma^{\prime} J_{\imath \jmath} \hat{\sigma}_{\imath} \cdot \hat{\sigma}_{\jmath}
$$

where $\hat{\sigma}_{\imath}$ is the pseudo-spin operator, the eigenvalues of which are +1 in the HS state, -1 in the LS state; $\Sigma$ sums over molecules and $\Sigma^{\prime}$ over pairs.

We stress on the phenomenological character of the Ising-like model. The interaction parameter has not been directly measured in any case; a single attempt has been made to predict its value by calculating the energies of a pair of molecules in different spin states: Molecular Orbital calculations have been presented in Bolvin's thesis [8] but they are not accurate enough to be really conclusive. Furthermore, it is not clear whether one can define a value of the interaction parameter between two neighbouring molecules, irrespective of the spin states of the surrounding molecules. We have undertaken the study of a diluted system in order to obtain some information concerning this difficult point.

From a general point of view, the theory of diluted systems was developed for magnetic systems, leading to the concept of percolation [9]. The value of the percolation threshold was found to be extremely sensitive to the interaction range (see [10] for example). Concerning diluted spin-crossover systems, a previous adaptation of the macroscopic elastic theory (infinite range interactions) was successful, particularly in the case of $\left[\left(\mathrm{Fe}_{x} \mathrm{Zn}_{1-x}\right)(2 \text {-pic) })_{3}\right] \mathrm{Cl}_{2}$.EtOH [11]. We use here an Ising-like approach solved by numerical simulations, in order to avold the infinite-range approximation involved in the mean-field approach. On the other hand. it has been shown that the numerical simulations based on the Monte Carlo Metropolis method better reproduce the shape of the hysteresis loops [12], which here corresponds to an important aspect of the avalable information (due to the quasi-complete character of the conversions).

The system $\left[\mathrm{Fe}_{x} \mathrm{Co}_{1-x}\left(4,4^{\prime}-\text { bis-1,2,4-triazole }\right)_{2}(\mathrm{NCS})_{2}\right] \cdot \mathrm{H}_{2} \mathrm{O}$, under study here, abbreviated as $\left[\mathrm{Fe}_{x}\right]$, has been studied over the whole composition range, by magnetic, calorimetric and Mössbauer measurements. The experimental data have been reported in [13], together with an interpretation in terms of a regular solution model, equivalent to an Ising-type mean-field approach. An essential feature is the decrease of the width of the hysteresis loop on decreasing $x$; the loop collapses below a threshold value $x_{\mathrm{c}} \approx 0.4$. This value is refined in the present work, thanks to new samples; all the data are summarized in Section 2, together with the calorimetric data which are re-examined. Section 3 is devoted to a brief recall of the Monte Carlo Metropolis technique, Section 4 to the results and discussion.

At the beginning of the present work, our idea was to investigate the range of the intermolecular interactions. The results were found to depend very little on the interaction range, but to sizeably differ from the experimental data. We finally found a convenient agreement by introducing "indirect" interactions between two crossover units via a non-spın-crossover unit, by purely formal analogy to magnetic super-exchange interactions. The physical meaning of such indirect interactions is discussed in steric terms. 


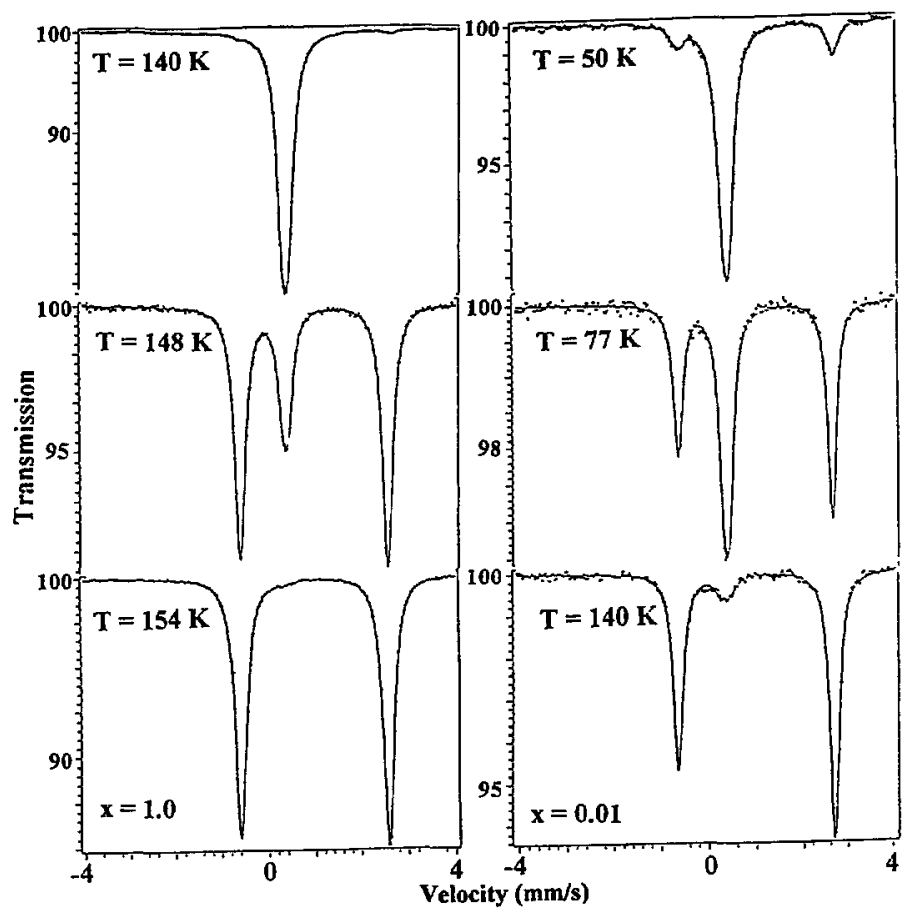

Fig. 1. - Selected Mössbauer spectra of $\left[\mathrm{Fe}_{1.0}\right]$ (in the heating mode) and [ $\left.\mathrm{Fe}_{0.01}\right]$.

\section{Experimental Data}

In the solid state $\left[\mathrm{Fe}_{x} \mathrm{Co}_{1-x}\left(4,4^{\prime}-\text { bis-1,2,4-triazole }\right)_{2}(\mathrm{NCS})_{2}\right] \cdot \mathrm{H}_{2} \mathrm{O}$, consists of layers of sixcoordinated metal ions linked to each other in the equatorial plane through the N1, N1'bridging triazole ligands, basically giving the system a $2 \mathrm{D}$-character. The iron sites form, at first approximation, a square planar lattice. Magnetic and Mössbauer measurements [13] show that the thermal hysteresis loop, $\approx 24 \mathrm{~K}$ wide for $x=1$, rapidly narrows on decreasing $x$, and collapses at the threshold value $x_{\mathrm{c}} \approx 0.37$. These data are summarised in Figures 1-4.

Selected ${ }^{57} \mathrm{Fe}$ Mössbauer spectra for $x=1$ and $x=0.01$ are shown in Figure 1 . At high temperature all Mössbauer spectra exhibit a single doublet with quadrupole splitting $(2.80-2.85 \mathrm{~mm} / \mathrm{s})$ and isomer shift $(1.04-1.05 \mathrm{~mm} / \mathrm{s}$, with respect to metallic iron at room temperature) values typical for high-spin (HS) $\mathrm{Fe}^{\mathrm{II}}$ At lower temperature a new peak appears with a very small quadrupole splitting $(0.11-0.15 \mathrm{~mm} / \mathrm{s})$ and isomer shift $(0.52-0.54 \mathrm{~mm} / \mathrm{s})$ values typical for low-spin (LS) $\mathrm{Fe}^{\mathrm{II}}$.

Typical conversion curves $n_{\mathrm{HS}}(T)$ are presented in Figure 2. The hysteresis width $\Delta T_{\mathrm{C}}$ is defined as $T_{\mathrm{C}} \uparrow-T_{\mathrm{C}} \downarrow$, where these temperatures, as well as $T_{1 / 2}$, are defined as the temperatures for which the spin transformation is half complete (i.e. for $n_{\mathrm{HS}}=1 / 2$ after correction for the residual HS and LS values). The whole set of data for these critical temperatures is presented in Table I.

We show in Figure 3 the Mössbauer data of the spin conversion in the diluted system, $x=0.01$, plotted in Arrhenius axes. To a first approximation, the plot is linear, which is typical for a spin equilibrium between independent spin-crossover units; the small deviation of the plot from linearity in the high-temperature region is consistent with a vibrational effect 


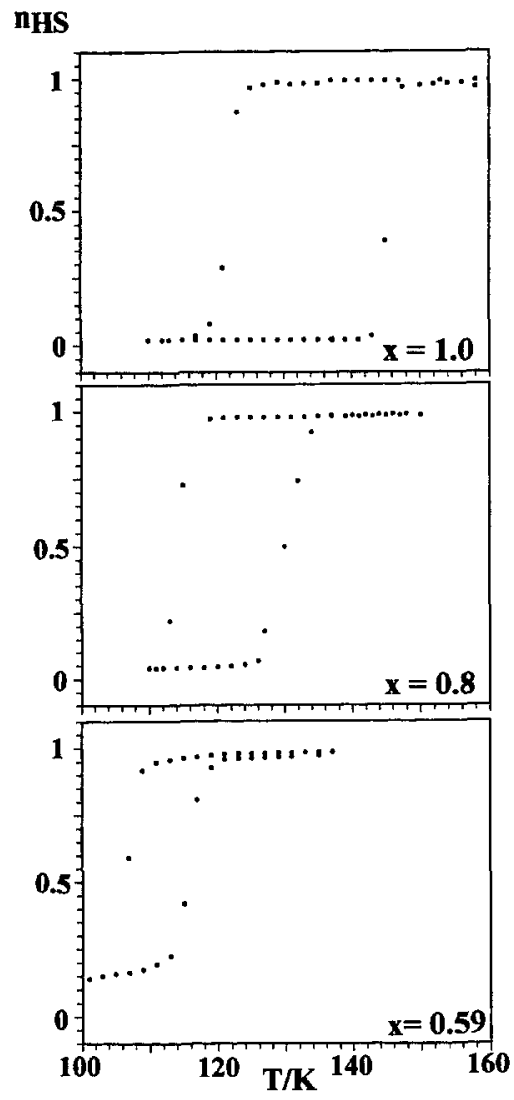

Fig. 2. - Thermal hysteresis loops of $\left[\mathrm{Fe}_{1}\right],\left[\mathrm{Fe}_{0.8}\right],\left[\mathrm{Fe}_{0.59}\right]$, from magnetic data [13].

(thermal population of different vibrational states in the LS, HS states [14]). A linear regression around the equilibrium temperature provides accurate determinations of $\Delta H, \Delta S$, which are reported in Table I.

In Figure 4 we summarize the variations of $T_{1 / 2}, T_{\mathrm{C}} \uparrow, T_{\mathrm{C}} \downarrow$ versus $x$, which form the phase diagram of the system in the $(T, x)$ space. It is worth noting the quite linear shape of the 2 branches for $x>x_{\mathrm{c}}$. Linear regressions give for all branches:

$$
\begin{array}{lll}
\text { for } & x<0.4 & T_{1 / 2} \sim 78+68 x \\
\text { for } \quad x>0.4: & T_{\mathrm{C}} \uparrow \sim 78.5+66 x ; & T_{\mathrm{C}} \downarrow \sim 92+29 x
\end{array}
$$

Equations $(2,3)$ form the borderlines of the "idealized" experımental phase diagram, and accurately determine the collapse value of the hysteresis loop: $x_{\mathrm{C}} \sim 0.36$.

In order to discuss the present data, it is worth considering an "equilibrium transition temperature", $T_{\text {eq }}$, in-between $T_{\mathrm{C}} \uparrow$ and $T_{\mathrm{C}} \downarrow$, corresponding to equal free energies to the HS and LS phases (then $x=1 / 2$ ). At $T_{\text {eq }}, \Delta H=T \Delta S$, giving similar thermodynamical properties to $T_{\text {eq }}\left(x>x_{\mathrm{c}}\right)$ and $T_{1 / 2}\left(x<x_{\mathrm{c}}\right)$; therefore the $T_{\text {eq }}(x)$ line is expected to exactly follow the $T_{1 / 2}(x)$ line. However, $T_{\text {eq }}$ cannot be measured from the experıments; as a first approach, we 


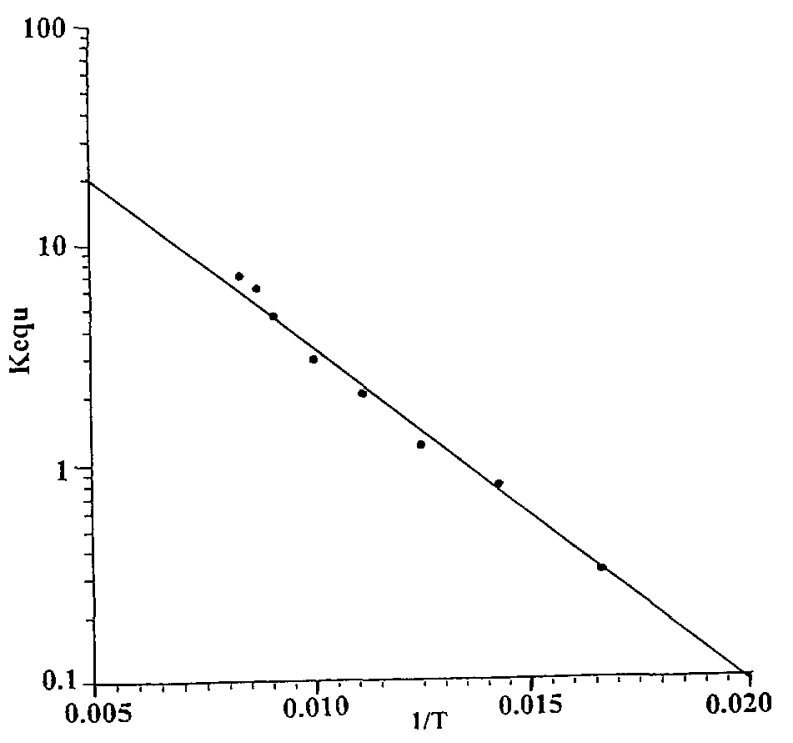

Fig. 3. - Arrhenıus plot of the spin equllibrium in [Fe.01], from the Mössbauer data, and best linear fit: equilibrium constant $K_{\text {eq }}=\frac{n_{\mathrm{HS}}}{1-n_{\mathrm{HS}}}$ versus $1 / T$.

Table I. - Experimental data for the equibrium and transition temperatures, molar enthalpy and entropy changes upon total conversion of $[\mathrm{Fe}]$ and ratio of the deduced effective degeneracies, for the system $\left[\mathrm{Fe}_{x} \mathrm{Co}_{1-x}\left(4,4^{\prime}-\text { bis-1,2,4-triazole }\right)_{2}(\mathrm{NCS})_{2}\right] . \mathrm{H}_{2} \mathrm{O}$.

\begin{tabular}{|c|c|c|c|c|c|c|}
\hline$x$ & $\begin{array}{c}T_{1 / 2} \\
(\mathrm{~K})\end{array}$ & $\begin{array}{c}T_{\mathrm{C} \uparrow} \\
(\mathrm{K})\end{array}$ & $\begin{array}{c}T_{\mathrm{C}} \downarrow \\
(\mathrm{K})\end{array}$ & $\begin{array}{c}\Delta H(\exp ) \\
\mathrm{kJ} \mathrm{mol}^{-1}\end{array}$ & $\begin{array}{c}\Delta S \\
\mathrm{~J} \mathrm{~K}^{-1} \mathrm{~mol}^{-1}\end{array}$ & $\mathrm{~g}$ \\
\hline 0.01 & 75 & & & 2.90 & 38.5 & 105 \\
0.05 & 82.3 & & & 3.25 & & \\
0.24 & 97 & & & 5.21 & & \\
0.39 & & 107 & 105 & 5.31 & 53.7 & 638 \\
0.54 & & 114 & 107 & 6.43 & 59.9 & 1346 \\
0.59 & & 115 & 107 & 7.60 & 61.8 & 1690 \\
0.8 & & 130 & 115 & 7.72 & 69 & 3995 \\
1.0 & & 145 & 121 & 10.20 & 74.8 & 8021 \\
\hline
\end{tabular}

took the following empirical relation (derived from numerical simulations [12]):

$$
T_{\text {eq }}=\frac{9 T_{\mathrm{C}} \uparrow+7 T_{\mathrm{C}} \downarrow}{16}
$$

leading to:

$$
T_{\text {eq }}(x)=84.4+49.8 x
$$

which is shown in Figure 4 as a dashed line.

As expected, the $T_{\text {eq }}$ line follows the $T_{1 / 2}$ line; the latter consists of a linear variation down to $x \approx 0.2$, with an extra-decrease for the most diluted samples. A similar trend has been observed on a $[\mathrm{Fe}, \mathrm{Zn}]$ system $[1 \mathrm{c}, 15]$, and we suggest, as a possible explanation, an isotopic 


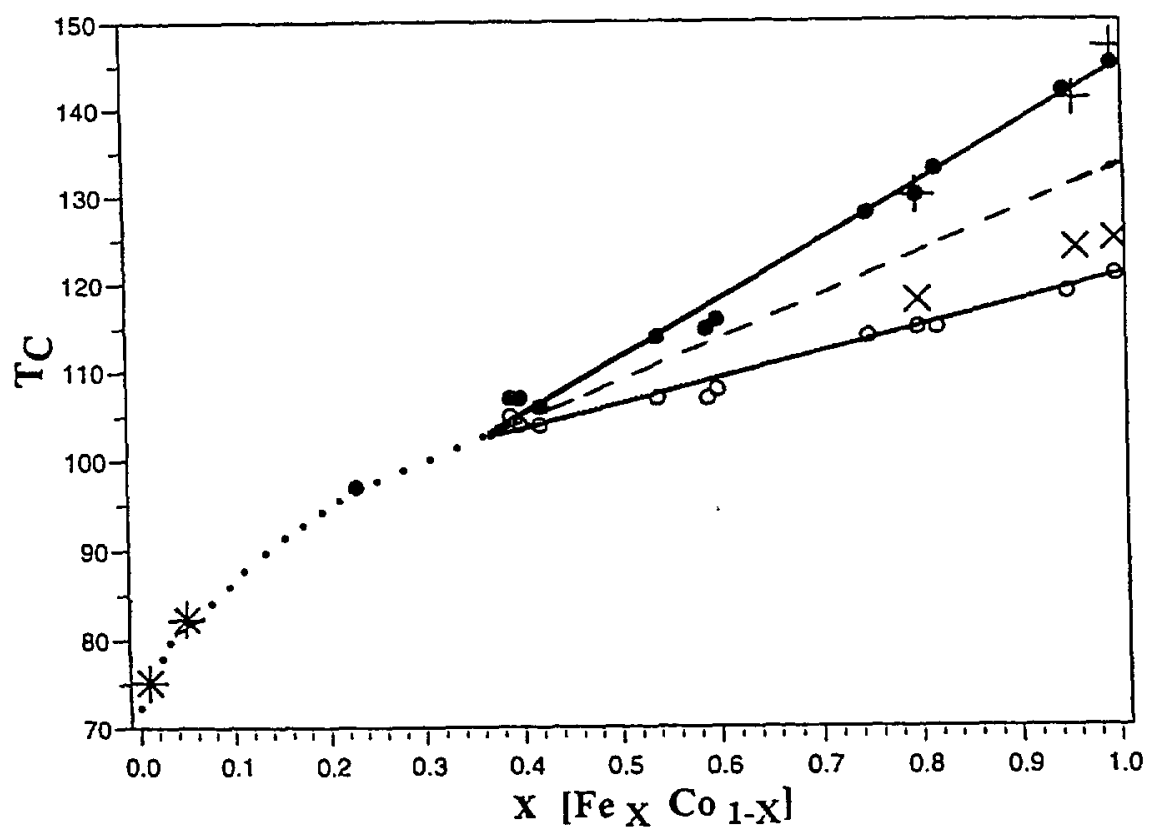

Fig. 4. - Collected data of $T_{1 / 2}, T_{\mathrm{C} \uparrow}$ on heating $(\bullet,+)$ and $T_{\mathrm{C} \downarrow}$ on cooling $(O, \times)$ modes derived from magnetic susceptibility $(\bullet, O)$ and Mössbauer measurements $(+, \times, *)$. Solıd lines are best linear fits used to determine the phase diagram of the system. $T_{\text {eq }}$ and $T_{1 / 2}$ are shown as a dashed and a dotted line, respectively.

effect associated with the ${ }^{57} \mathrm{Fe}$-enriched character of the most diluted samples. Such an effect, if confirmed, might lead to reconsıder (slightly) all Mössbauer data in the spin equilibrium literature.

We have also re-examined the calorimetric data given in [13]. The point is that the measured $\Delta H$ values are the result of integrations over rather narrow temperature ranges, compared to the spin conversion ranges obtained from Mössbauer and magnetic susceptibility measurements, and therefore refer to the partial conversion of one iron mole in the sample. So these data have to be corrected accordingly:

$$
(\Delta H)_{\text {corrected }}=(\Delta H)_{\text {measured }} / \Delta n_{\mathrm{HS}}
$$

where $\Delta n_{\mathrm{HS}}$ is estimated from the magnetic and Mössbauer data.

Corrected values are given in Table I, and plotted in Figure 5 versus composition. The correction introduces some uncertainties, mostly for small Fe concentrations, but now the general trend perfectly includes the Mössbauer data of the most diluted systems. We have also smoothed the $\Delta H(x)$ data, using:

$$
\Delta H(x)=2800+7260 x
$$

The $\Delta S(x)$ values have been deduced from the equilibrium relation:

$$
\Delta H(x)=T_{1 / 2}(x) \Delta S(x)
$$

where $T_{1 / 2}, T_{\text {eq }}$ were taken from equations $(2,5)$ respectively. 


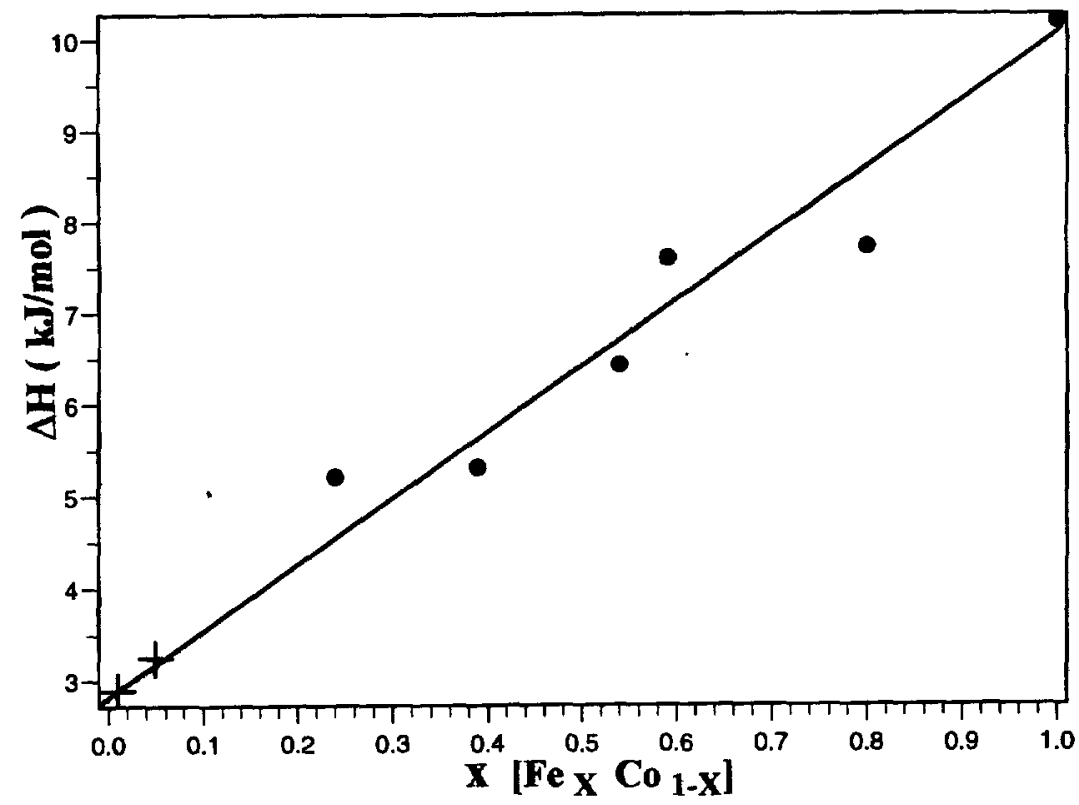

Fig. 5. - Enthalpy change upon spin conversion for an iron(II) mole, deduced from calorimetric (•) and Mössbauer data $(+)$.

These thermodynamic data provide the input parameters of the Ising-like Hamiltonian, as follows:

energy gap : $\Delta(x)=\Delta H(x) / \mathcal{N}$, where $\mathcal{N}$ is Avogadro's number; (9)

"effective" degeneracy ratio : $\quad g(x)=g_{\mathrm{HS}} / g_{\mathrm{LS}}=\exp (\Delta S(x) / R)$

The sizeable dependence of these parameters upon composition can be briefly discussed:

(i) $\Delta(x)$ can be related to the lattice parameter variation, as previously discussed in [13]: introducing Co units in the Fe lattice probably results in an average increase of the lattice parameters (averaged over the LS, HS phases), because the lonic radius of HS CoII is larger than the average LS-HS Fe $\mathrm{I}^{\mathrm{II}}$ radius: respectively $0.88 \AA$ and $(0.75+0.92) / 2=$ $0.83 \AA$. The increase of the lattice parameter induces a "negative pressure" on the spincrossover unit, thus favouring the HS state, i.e. lowering the transition temperature. The same explanation holds for the [Fe, $\mathrm{Zn}$ ] system of references [1c, 15]. On the contrary, in the [Fe, $\mathrm{Ni}$ ] system isomorphous to the present one [16], the average transition temperature remains remarkably constant, because the $\mathrm{HS} \mathrm{Ni}^{\mathrm{II}}$ ionic radius exactly matches the average $\mathrm{HS}-\mathrm{LS} \mathrm{Fe}^{\mathrm{II}}$ radius;

(ii) the large composition dependence of $\Delta S(x)$ has been emphasized in [13] and described in terms of a vibrational contribution, which is highly composition dependent. It implies that some of the $[\mathrm{Fe}]$ unit vibrations are sensitive to the nature of the surrounding units. In other words, these internal modes are coupled to the external modes of the units. In line with this idea, we already suggested in our electrovibrational model [14] that the effective degeneracies of the two states might depend on the spm state of the surroundings (thus leading to cooperative entropic effect). 


\section{Monte Carlo - Metropolis Simulations}

The Metropolis algorithm provides the most representative configurations of the thermodynamical system through a Markov chain of elementary transformations yielding a limit which corresponds to the canonical equilibrium distribution. For this purpose the equilibrium distribution $P(i, T) \propto \mathrm{e}^{-\beta E_{2}}$ is used in the detailed balance equation.

$$
P(i, T) W(i \rightarrow j, T)=P(j, T) W(j \rightarrow i, T)
$$

For the practical aspects, the Metropolis algorithm [17] is the following: consider an initial configuration $A_{\imath}$ with energy $E_{\imath}$. First a lattice site $j$ is selected at random and the energy $E_{\mathrm{f}}$ is calculated when $\sigma_{\jmath}$ is flipped. Then the transition probability $w_{\sigma_{\jmath} \rightarrow-\sigma_{\jmath}}=\mathrm{e}^{-\beta\left(E_{\mathrm{f}}-E_{2}\right)}$ is computed. Next a random number between 0 and unity is generated to be compared with the transition probability. If the probability $w_{\sigma_{3} \rightarrow-\sigma_{3}}$ is larger than the random number the new configuration is accepted. Otherwise the lattice site remains with the initial value $\sigma_{3}$. This procedure, as explained in [17], obeys the detailed balance equation and provides the fastest convergence of iterative process. The algorithm continues by selecting another lattice site.

To account for the different degeneracies $g_{\mathrm{HS}}, g_{\mathrm{LS}}$, we have introduced a pre-exponential factor [12], thus leading to the completed expression:

$$
w_{\sigma_{3} \rightarrow-\sigma_{3}}=\frac{g_{-\sigma_{3}}}{g_{\sigma_{3}}} \mathrm{e}^{-\beta\left(E_{\mathbf{f}}-E_{2}\right)}
$$

where the pre-exponential factor $g_{-\sigma_{\imath}} / g_{\sigma_{2}}$ takes the values $g$ for LS $\rightarrow$ HS and $1 / g$ for HS $\rightarrow$ LS.

For calculations the square lattice was $100 \times 100 ; 2000$ Monte Carlo steps were performed for reaching the thermal equilıbrium, and again 2000 for computing the average values. Temperature steps were typically $1 \mathrm{~K}$.

\section{Results and Discussion}

In Figure 6 we show the results obtained with only nearest-neighbour interactions. For 2D interactions the computed phase diagram significantly departs from the experimental diagram: the $T_{\mathrm{C}} \uparrow, T_{\mathrm{C}} \downarrow$ borderlines are strongly curved; the collapse value of the hysteresis loop, $x_{\mathrm{c}} \sim 0.55$, is too large. In the percolation description, one would expect the hysteresis width $\Delta T_{\mathrm{C}}$ to decrease faster and faster with $x$ on approaching the threshold value. The computed data follow an opposite trend.

To improve the model, we have examined several possıbilities:

(i) $3 \mathrm{D}$ interactions. These case the results reported in Figure 6; the computer threshold value is still $x_{\mathrm{c}} \sim 0.55$ and the borderlines remain curved;

(ii) a composition-dependent interaction parameter $J(x)$ (as basically done in [13]): the needed variation, shown in Figure 7, is strongly non-linear and therefore seems unlikely, because all measured thermodynamical quantities vary quasi-linearly upon $x$;

(iii) further interactions, namely second-nearest-neighbour interactions: the results, reported in Figure 8, do not markedly differ from those obtained with nearest-neighbour;

(iv) indirect interactions through the Co units, of ferromagnetic type $(J>0)$, in order to provide an extra-contribution to cooperativity related to the presence of Co units. The pathways of these interactions is schematized in Figure 9. The computer program, already 


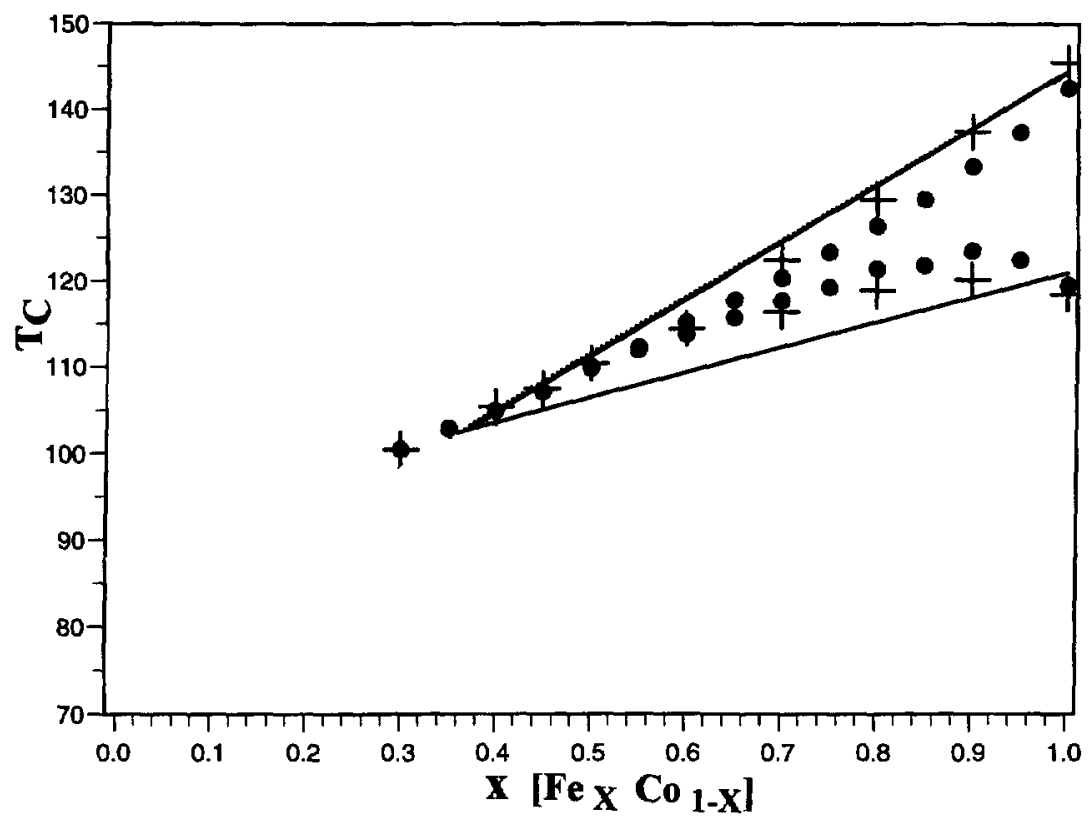

Fig. 6. - Experımental data (solıd lines) and Monte Carlo sımulation results with: nearest-neıghbour interactions ("direct" interaction), in 2D (•) and 3D (+) lattices; input parameters are listed in Table II.

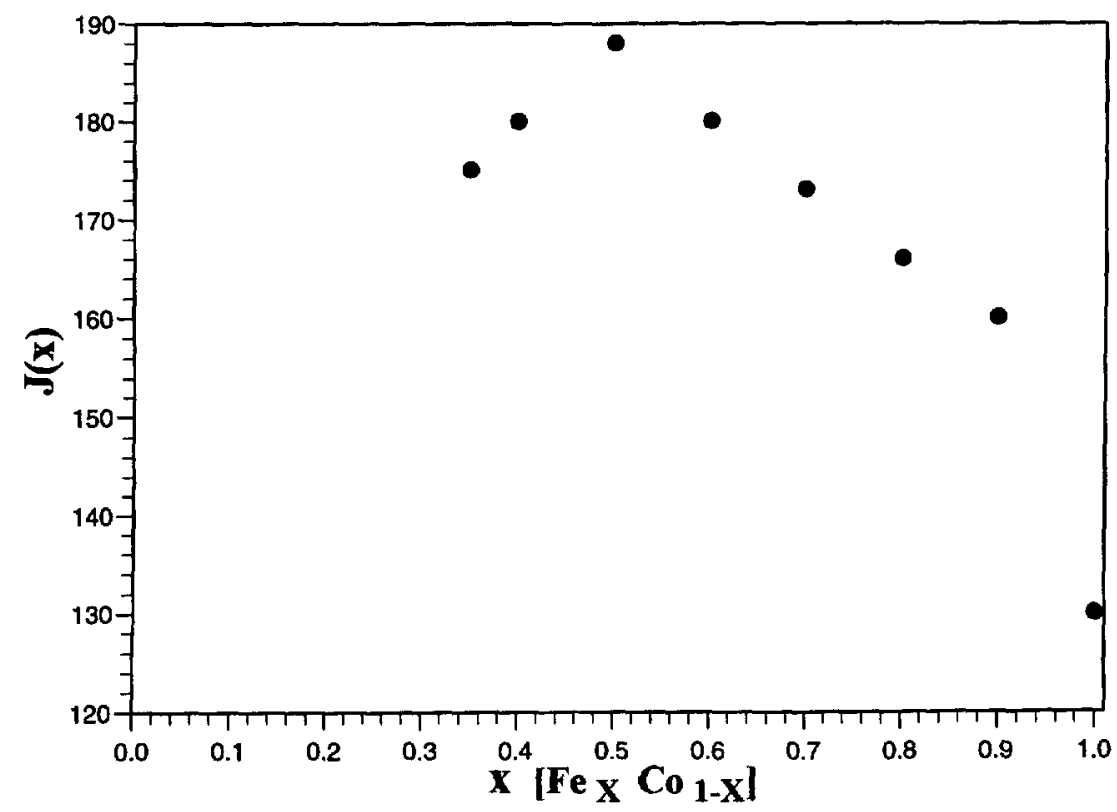

Fig. 7. - Composition dependence of the nearest-neighbour Fe-Fe interaction needed to reproduce the experimental data (1n 2D simulations). 


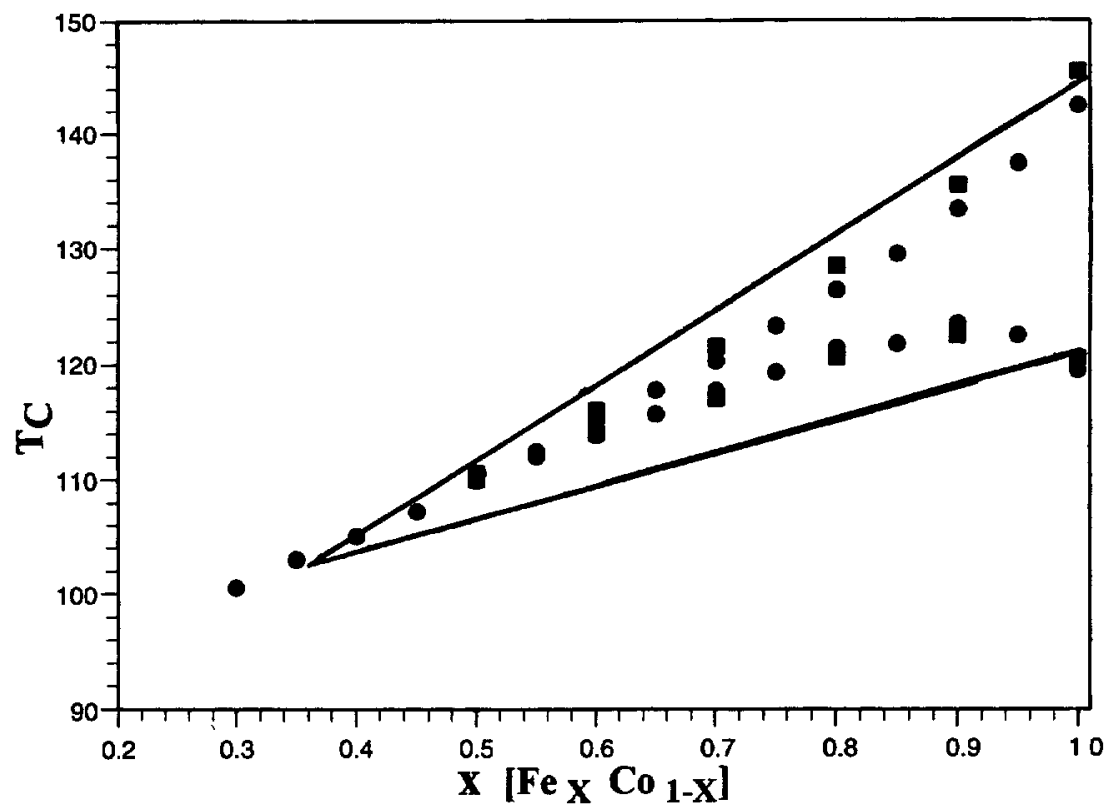

Fig. 8. - Numerical 2D simulations, comparing first-(•) and second-( $\boldsymbol{\square})$ nearest-neighbour direct $\mathrm{Fe}-\mathrm{Fe}$ interactions; input parameters are listed in Table II.

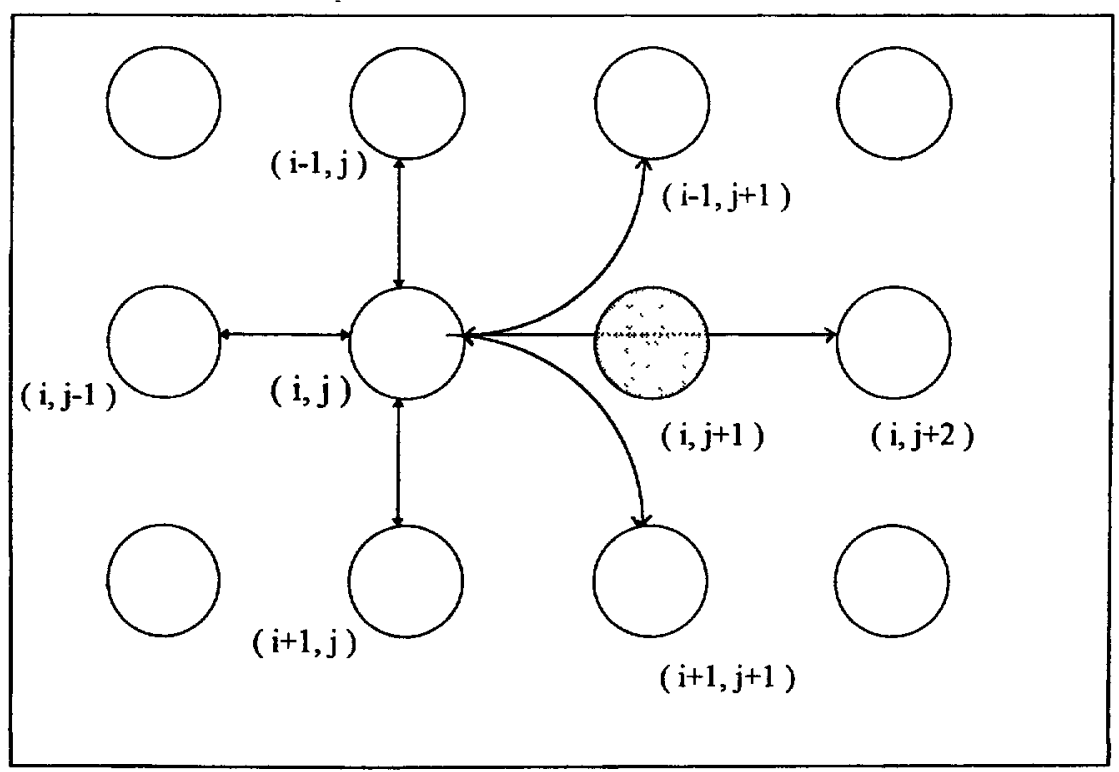

Fig. 9. - The pathways of direct $\mathrm{Fe}-\mathrm{Fe}$ and indirect $\mathrm{Fe}-\mathrm{Co}-\mathrm{Fe}$ interactions between two $\mathrm{Fe}$ units. Open circles stand for $\mathrm{Fe}$ units and closed circles for Co units. 


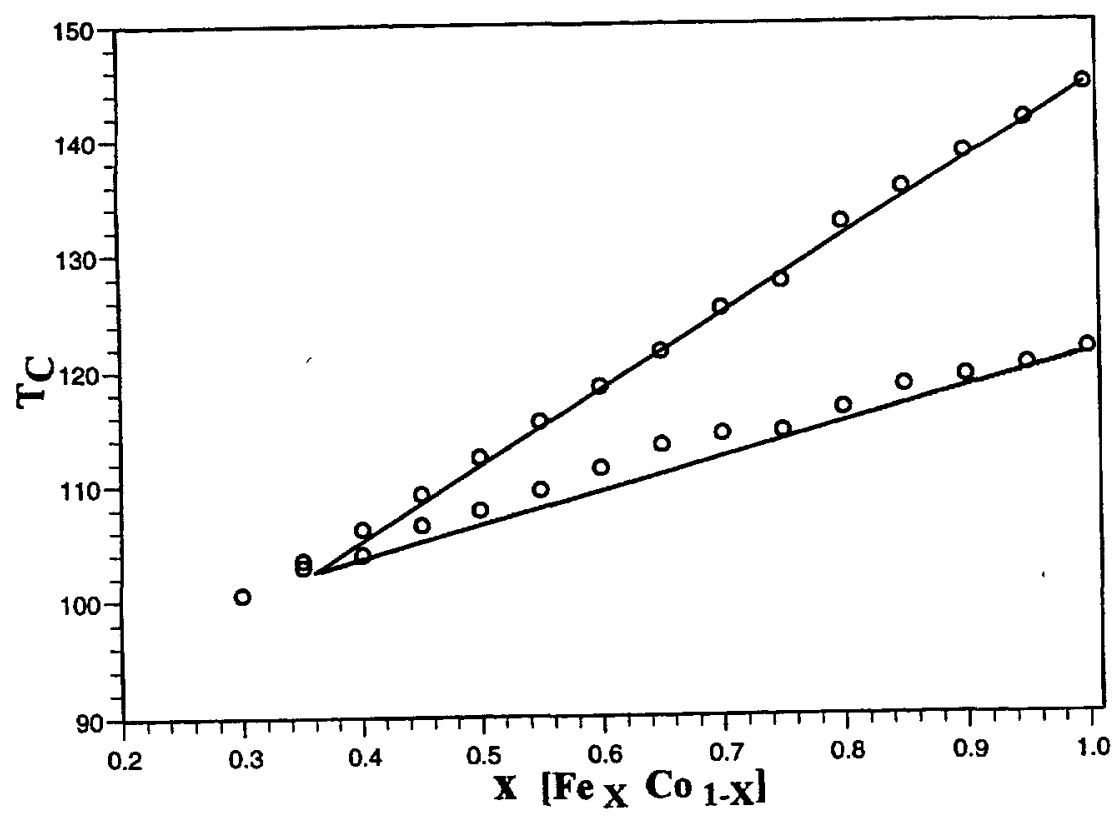

Fig. 10. - Numerical 2D simulations (O), including both direct $\mathrm{Fe}-\mathrm{Fe}$ and indirect FeCoFe interactions; input parameters are listed in Table II.

Table II. - Interaction parameter values $(K)$ for the numerical simulations.

\begin{tabular}{|l|r|r|r|l|}
\hline direct & $\mathrm{FeCoFe}$ & $\mathrm{Fe} \mathrm{Fe}^{(\mathrm{HS}, \mathrm{LS})} \mathrm{Fe}$ & Figure & Lattice (Comment) \\
\hline 1rst (2nd) & & & & \\
120 & & & $6,8(\bullet)$ & $2 \mathrm{D}$ \\
60 & & $6(+)$ & $3 \mathrm{D}$ \\
$100(20)$ & & & $8(\mathbf{\square})$ & $2 \mathrm{D}$ \\
125 & +28 & & $10(\mathrm{O})$ & $2 \mathrm{D}$ (good fit) \\
120 & & $-5^{(\mathrm{HS})}+10^{(\mathrm{LS})}$ & $11(\mathbf{\Delta})$ & $2 \mathrm{D}$ \\
50 & & $+16^{(\mathrm{HS})}+16^{(\mathrm{LS})}$ & $11(*)$ & $2 \mathrm{D}$ \\
\hline
\end{tabular}

developed for the second- and third-nearest-neighbour interactions, was easily adapted thanks to simple Kronecker functions. An excellent agreement was obtained with the experimental phase diagram, as shown in Figure 10. Parameter values are given in Table II. 


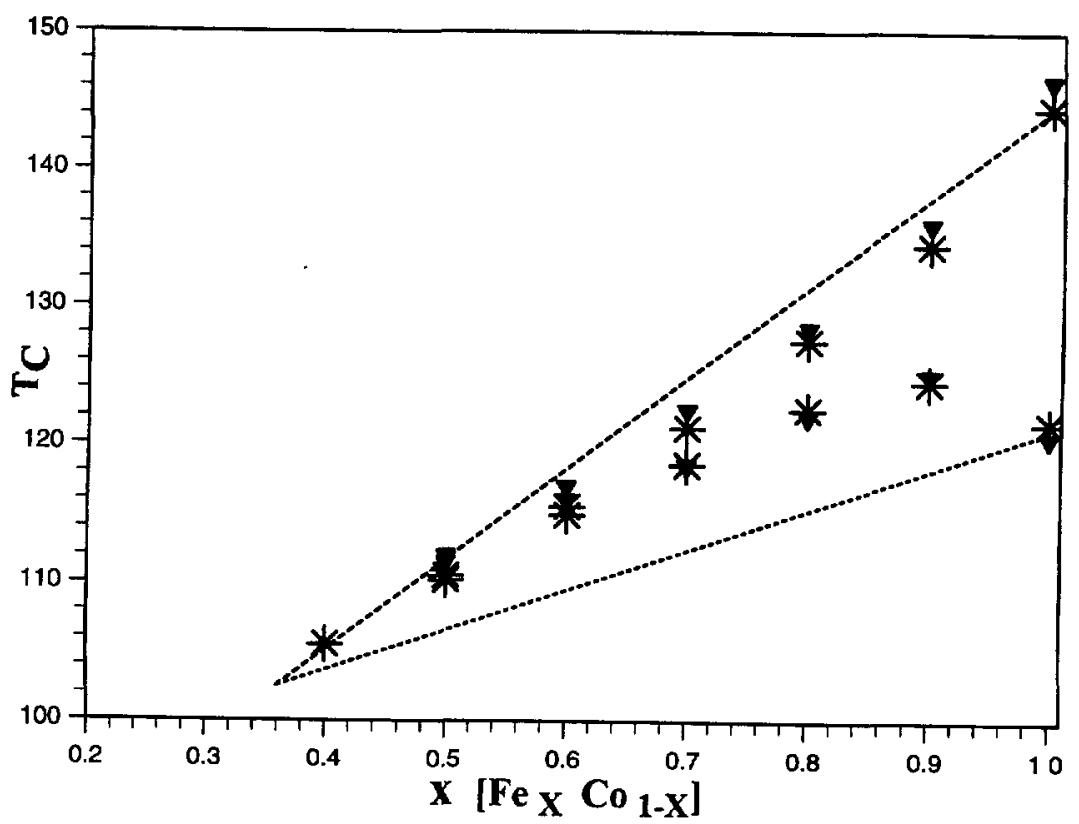

Fig. 11. - Numerical 2D simulations including both direct $\mathrm{Fe}-\mathrm{Fe}$ and indirect FeFeFe interactions; symbol definitions and input parameters are given in Table II.

Table III. - Computed data for the short range effect around Co units and vacancies: $n_{\mathrm{HS}, \mathrm{Co}}\left(n_{\mathrm{HS}, \mathrm{v}}\right)$ is the ratio of Fe units having at least a Co (vacancy) unit in a nearest-neighbour position. The rate of $C o$ and vacancies units was fixed to $10 \%$.

\begin{tabular}{|c|c|c|c|}
\hline$n_{\mathrm{HS}}$ & $n_{\mathrm{HS}, \mathrm{Co}}$ & $n_{\mathrm{HS}, \mathrm{v}}$ & $T(\mathrm{~K})$ \\
\hline 0.9970 & 0.9965 & 0.9927 & $139\left(7.5\right.$ above $\left.T_{\mathrm{c}} \uparrow\right)$ \\
0.0019 & 0.0039 & 0.0045 & $113\left(7.5\right.$ below $\left.T_{\mathrm{c}} \downarrow\right)$ \\
\hline
\end{tabular}

Such hypothetic indirect interactions have to be discussed at varıous levels:

(i) Similar Fe-Fe-Fe interactions might be introduced as well. We investigated two limiting cases: indirect interaction Fe-Fe-Fe irrespective of the spin state of the intermediate $\mathrm{Fe}^{\mathrm{II}}$, and interactions of opposite signs for $\mathrm{Fe}-\mathrm{Fe}^{\mathrm{HS}}-\mathrm{Fe}$ and $\mathrm{Fe}-\mathrm{Fe}^{\mathrm{LS}}-\mathrm{Fe}$. Results are shown in Figure 11 and listed in Table II. We observed in all cases that the indirect $\mathrm{Fe}-\mathrm{Fe}-\mathrm{Fe}$ interactions act in a way exactly simllar to the direct $\mathrm{Fe}-\mathrm{Fe}$ interaction. In other words, it is not possible to determine separately these $\mathrm{Fe}-\mathrm{Fe}$ and $\mathrm{Fe}-\mathrm{Fe}$-Fe interactions from the knowledge of the phase diagram. Interestingly, it appears that the system is not equally sensitive to the indirect interactions through the LS and HS Fe units: for example, a given interaction through a LS unit is balanced by a smaller (and opposite) interaction through a HS unit.

(ii) On the other hand, a qualitative explanation for the presence of such an indirect interaction through the Co units can be given in terms of steric effects. The effect of a few Co atoms introduced into the Fe lattice can be considered as follows; a long-range effect 
decreases the equilibrium temperature (as shown in Sect. 2); a short-range effect will differ according to the spin state of the solid: in the LS phase, around a (bigger) Co unit, a cluster of LS Fe units is stabilized; in the HS phase, around a (smaller) Co unit, a cluster of HS Fe units is also stabilized. Such an enhancement of the short-range correlations around the impurity unit is phenomenologically accounted for by the indirect "ferromagnetic- type" interaction. In Table III we selected computed data comparing the surroundings of Co units and of vacancies, which mimic Co units when the indirect interactions are switched off; the average $n_{\mathrm{HS}}$ value of Fe units surrounding the Co units departs more from the equilibrium value $(1 / 2)$ than that of $\mathrm{Fe}$ units surrounding the vacancies: the expected enhancement of the "ferromagnetic-type" correlation is indeed obtained.

We do not discuss here the direct interactions in similar steric terms. This remains an open problem, for which we have provided here a further piece of information, i.e. the weak influence of the range assumed for these phenomenological interactions.

At last, we briefly discuss the present study in terms of geometrical percolation: indeed, the introduction of Fe-Co-Fe interactions creates new paths for propagating the interactions, and lowers the hysteresis loop collapse value, $x_{c}$, in agreement with the expected decrease of the geometrical percolation threshold, $x_{\mathrm{g}}$. However, the computed $x_{\mathrm{c}}$ values are almost unsensitive to the dimensionality of the system, as well as to the range of the Fe-Fe interactions, at variance from $x_{\mathrm{g}}$ values. Obviously, the relationship between $x_{\mathrm{c}}$ and $x_{\mathrm{g}}$ is not tight, and certainly deserves a further investigation.

\section{Conclusion}

The effect of the dilution of a spin-crossover system has been studied in terms of the two-level model with nearest neighbours interactions, completed by an indirect interaction occurring via the non spin-crossover (Co) atom. The data are successfully reproduced and a steric explanation for the onset of these indirect interactions has been given.

\section{References}

[1] (a) Slichter C.P. and Drickamer H.G., J. Chem. Phys. 56 (1972) 2142; (b) Purcell K.F. and Edwards M.P., Inorg. Chem. 23 (1984) 2620; (c) Gütlich P., Köppen H., Link R. and Steinhäuser H. G., J. Chem. Phys. 70 (1979) 3977.

[2] (a) Spiering H. and Willenbacher N., J. Phys. Cond. Matter 1 (1989) 10089; (b) Willenbacher N. and Spiering H., J. Phys. C. Solid State Phys. 21 (1988) 1423.

[3] Bousseksou A., Nasser J., Linares J., Boukheddaden K. and Varret F., J. Phys. I France 2 (1992) 1381-1403.

[4] (a) Wajnflasz J., Phys. Stat. Solidi 40 (1970) 537; (b) Wajnflasz J. and Pick R., J. Phys. IV France 32 (1971) C1-91; (c) Bari R. and Sivardière J., Phys. Rev. B 5 (1972) 4466.

[5] Zimmermann R. and König E., J. Phys. Chem. Solids 38 (1977) 779.

[6] Nasser J. and Linares J., private communication.

[7] (a) Bolvin H. and Kahn O., Chem. Phys. 243 (1995) 355; (b) Linares J., Shteto I. and Varret F., in preparation.

[8] Bolvin H., PhD Thesis, Université de Paris-Sud (1993).

[9] Kirkpatrick S., Rev. Mod. Phys. 45 (1973) 574. 
[10] Stauffer D. and A. Aharony, Introduction to Percolation Theory, 2nd ed. (Taylor and Francis, London, 1994).

[11] Spiering H., Meissner E., Köppen H., Müller E.W. and Gütlich P., Chem. Phys. 68 (1982) 65.

[12] Linares J., Nasser J., Bousseksou A., Boukheddaden A. and Varret F., J. Magn. Magn. Mater. 140-144 (1995) 1507.

[13] Martin J.P., Zarembowitch J., Bousseksou A., Dworkin A., Haasnoot J.G. and Varret F., Inorg. Chem. 33 (1994) 6325-6333.

[14] Bousseksou A., Constant-Machado H, and Varret F., J. Phys. I France 5 (1995) 747-760.

[15] Sorai M., Ensling J. and Gütlich P., Chem. Phys. 18 (1976) 199.

[16] Martin J.P., Zarembowitch J., Dworkin A., Haasnoot J.G. and Codjovi E., Inorg. Chem. 33 (1994) 2617.

[17] Metropolis N., Rosenbluth A.W., Rosenbluth M.N., Teller A.H. and Teller E., J. Chem. Phys. 21 (1953) 1087. 